This article has been published in Electrochemica Acta. The final publication is available at Elsevier via https://doi.org/10.1016/j.electacta.2014.11.143.

\title{
X-ray Tomographic Analysis of Porosity Distributions in Gas Diffusion Layers of Proton Exchange Membrane Fuel Cells
}

\author{
S. Odaya ${ }^{1}$, R.K. Phillips ${ }^{1}$, Y. Sharma ${ }^{1}$, J. Bellerive ${ }^{2}$, A.B. Phillion ${ }^{1}$, \& M. Hoorfar ${ }^{1}$ \\ ${ }^{1}$ School of Engineering, University of British Columbia, 3333 University Way, Kelowna, BC \\ V1V 1V7, Canada \\ ${ }^{2}$ Ballard Power Systems, 9000 Glenlyon Parkway, Burnaby, BC, V5J 5J8, Canada
}

\section{Abstract}

This paper describes a method to characterize the structure of polytetrafluoroethylene (PTFE) treated gas diffusion layers (GDLs) with and without microporous layers (MPLs) using 3D X-ray micro computed tomographic $(\mu \mathrm{CT})$ microscopy. In this work, the structure of single and dual layer GDLs is evaluated via $\mu \mathrm{CT}$ for various GDL samples (such as Toray TGP-H-060 and AvCarb EP40) loaded with different MPLs. A new method is presented for separating, or segmenting, the various phases of the GDL, i.e., void space, carbon fiber (including binder and PTFE), and MPL. Through analysis, it was found that the variation in bulk porosity and the average pore diameter of the GDLs depends highly on the GDL series manufacturing and treatment processes. Using advanced image analysis techniques, routines were developed to accurately segment the GDL fibers (including binder/PTFE) and the MPL. The percentage of the intruding MPL material into the carbon fiber paper as a function of the GDL thickness was successfully found for dual layer GDLs, with varying PTFE content and areal weight loading in the MPL. This analysis provides invaluable insight into the physical microstructure of paper-based GDLs, emphasizing the heterogeneous porosity distribution of single layer GDLs and the interaction of the MPL with the carbon fiber paper of dual layer GDLs.

\section{Keywords}

Proton exchange membrane fuel cell, Gas diffusion layer, X-ray Computed Tomography, Porosity, Image analysis 


\section{Introduction}

The proton exchange membrane fuel cell (PEMFC) has recently received significant attention from the automotive industry as a clean and efficient energy system with a significant potential for development and integration into transportation systems [1-3]. A PEMFC creates electrical power through the electrochemical reduction-oxidation reaction of a fuel and an oxidant. Commonly, hydrogen gas is used as a fuel at the anode and oxygen gas (or air) is used as an oxidant at the cathode, with only heat and water as by-products of the reaction. The anode and cathode electrodes each consist of a catalyst layer (CL), a gas diffusion layer (GDL), and a bipolar plate. The electrodes are separated by a perfluorosulfonic acid (PFSA) membrane that allows for proton exchange from the anode to the cathode, while electron transfer occurs through an external load. The PFSA membrane, layered with a CL and GDL on both sides, forms the membrane electrode assembly (MEA), which is the core of a PEMFC. Reactant gases are transported through a flow field in the bipolar plate to their respective electrodes of the MEA and are then diffused through the GDLs to the CLs, which is where the reduction-oxidation reaction occurs. The ineffective transport of reactants to, and removal of products from, the reaction sites on the CLs can lead to mass transport issues within the cell which, in turn, lead to decreased performance, especially in high power density regions $[3,4]$.

The GDL of a PEMFC is an integral layer which enhances the diffusion of reactant gases from the flow field to the CL of the respective electrodes [5, 4]. This porous carbon layer is important for the electrical connection and heat dissipation between the CL and bipolar plate; while it also acts as a mechanical support for the MEA [3]. Another key process occurring in the GDL is the mitigation of the water produced at the cathode by the electrochemical reaction [6]. In essence, the GDL must remove excess water to avoid flooding of the electrodes, while the PFSA membrane 
must be kept well hydrated to maintain high proton conductivity [3]. To ensure this proper water saturation balance, it is necessary to understand the structural properties and the two-phase flow of reactant gas and water within the GDL to help with mitigation strategies for improving mass transport properties $[6,3]$.

The GDL is composed of carbon fiber strands (dia. $\sim 7 \mu \mathrm{m})$ manufactured into a sheet-like product [7]. The fuel cell community commonly uses three different GDL types: paper-, felt-, and cloth-based. The main difference between these GDLs is the mechanisms in which the carbon fibers are held together in the manufacturing process, as described in detail by Mathias et al. [7]. Paper-based GDLs consist of carbon fibers, machine laid and bound together by a chemical binder. This is followed by impregnation of a carbonizable thermoset resin and a heat-treatment process for carbonization/graphitization of the resin and carbon fibers. The dried resin acts as a binder to hold the carbon fibers together and the heat-treatment process helps to improve electrical and wetting properties. Another approach for paper-based GDLs is to add a carbon or graphite powder to the resin binder in the paper making process to help further improve the electrical properties and also impart hydrophobic properties to the material [7].

To ensure that pores do not become flooded with liquid water, leading to impeded gas diffusion, GDLs are commonly treated with hydrophobic coatings such as polytetrafluroethylene (PTFE) and/or microporous layers (MPLs). The untreated (unteflonated) or treated (teflonated) macroporous carbon fiber paper substrate is referred to as a single-layer GDL, while a GDL with an MPL is referred to as a dual layer GDL [4]. To increase the hydrophobicity of single layer GDLs, the paper is commonly impregnated with an aqueous PTFE dispersion, followed by a drying and heat-treatment process to remove the remaining solvent, and also fix the PTFE and carbonize the constituents [7]. For dual layer GDLs, the MPL consists of a carbon black powder and a 
hydrophobic agent (commonly PTFE) applied to one or both sides of the carbon fiber substrate and taken through a further drying and heat treatment process [7]. The addition of an MPL serves to improve water management and electrical contact between the GDL and CL $[2,4]$. Together, the addition of these coatings has been repeatedly proven to significantly increase PEMFC performance, especially in regions of high current densities [4, 5, 8-10].

A number of recent studies have provided new insight into the physical structure of the GDL. The chief metrics for characterization are related to the pore size distribution (PSD) and bulk porosity, since these features directly affect the GDL's mass transport properties [11]. There are two main methods used to characterize these properties: mercury intrusion porosimetry (MIP) and capillary flow or method of standard porometry (MSP). MIP provides volumetric information about the PSD of the GDL, while MSP determines the PSD of only the smallest diameter of a tortuous path (throat of through-pore) within the GDL. As mentioned in Arvay et al. [3], MSP may serve as a better method for measuring PSD than MIP since it uses a much lower pressure and therefore has less chance of distorting pores or destroying the GDL sample $[3,11]$. A typical graph of the PSD measurement of a GDL reveals a bimodal distribution with micropores and macropores. The micropores can be explained by voids formed in the hydrophobic coatings (i.e., voids in the binder/PTFE or voids between carbon nanoparticles within the MPL); whereas the macropores represent the pores formed between the GDL carbon fiber strands or large cracks within the MPL $[8,9,3]$.

Han et al. [8] combined Scanning-Electron Microscopy (SEM) and MIP to characterize the pore structure of single layer Toray TGP-H-030 GDLs with $20 \mathrm{wt} \%$ PTFE content. The authors found a bimodal PSD with a smaller peak at $85 \mathrm{~nm}$ (representing micropores formed by small agglomerates within the binder and PTFE) and a larger and broader peak at $40.3 \mu \mathrm{m}$ (representing 
macropores formed by the carbon paper) [8]. This study also looked at embedding the carbon paper with a mixture of carbon particles and PTFE, which was called a carbon-filled GDL (CFGDL). Their results showed that the total porosity of single layer and CFGDLs were $77 \%$ and $67 \%$, while the average pore diameters were 35.8 and $4.7 \mu \mathrm{m}$. In-situ testing confirmed an increase in cell performance using the CFGDL as compared to the single layer GDL, especially around limiting current densities. The authors attributed this increase to the preferential formation of micro-water droplets within the modified microstructure of the CFGDL, which reduces mass transport losses [8]. This study demonstrates the importance of the optimization of the GDL parameters for maximum fuel cell performance.

Phillips et al. [12] characterized untreated and treated, single layer paper-based GDLs through the ex-situ measurement of transport properties such as wettability, pore size distribution (PSD), and permeability. Using MSP, it was found that the average pore diameters for the samples of Toray TGP-H-060 with PTFE levels of 0, 6, 19 wt\% decreased only slightly from 33, 30.5, $29 \mu \mathrm{m}$, respectively. Based on these results [12] and those presented in [7, 8-10, 13], it has been found that the amount of PTFE applied affect several mass transport characteristics within PEMFCs, and can greatly enhance their performance.

Parikh et al. [11] used image analysis techniques to obtain a PSD from 2D SEM images for different single layer GDL types (Freudenberg H2315 non-woven, SGL 25 BC with 5\% PTFE, and TGP-H-060 with 7\% PTFE). They found a considerable variation in the pore sizes, shapes and clustering between different GDLs, with the average pore size ranging from $16.5 \mu \mathrm{m}$ for the Freudenberg, $31.83 \mu \mathrm{m}$ for the SGL, to $26.4 \mu \mathrm{m}$ for Toray. These results are in good agreement with the results obtained from MIP and MSP techniques $[11,8,9,12]$. In an effort to understand the 3D structure of the GDL, a stochastic model has been used in [11] to construct a 3D realization of the 
studied GDLs based on the 2D SEM images and pre-determined pore parameters. The authors suggest that the 3D geometry should be used in modeling to yield a more representative behavior of the two-phase flow in the GDL. Additionally, they proposed that the GDLs microstructural properties should not solely be characterized by PSD, but also by pore shape and clustering of pores in the interconnected porous network of the GDL [11].

The above studies utilize bulk methods (i.e. MIP and/or MSP), along with 2D SEM imaging, to characterize the porosity and PSD of GDLs. However, it is inherently known that GDLs have a complex porous 3D structure that can vary in the in-plane (IP) and through-plane (TP) directions. High-resolution X-ray computed tomographic microscopy $(\mu \mathrm{CT})$ techniques have been increasingly utilized in recent years as a valuable tool for visualizing and understanding the complex 3D microstructure of the GDL.

In a primary study, Sinha et al. [14] used $\mu \mathrm{CT}$ to image the liquid water distribution in a GDL, and thus to obtain a liquid saturation curve across the GDL thickness. Further, Buchi et al. $[15,16]$ have used $\mu \mathrm{CT}$ to obtain the local water saturation level across the GDL thickness for different water pressures. Other similar studies have proven $\mu \mathrm{CT}$ to be useful in the imaging and analysis of the water configuration within the GDL $[6,17,18]$. Using $\mu \mathrm{CT}$, Markotter et al. [19, 20] have shown vivid visualizations of the water transport paths in the GDL with an in-situ PEMFC setup after ceasing the reactant flow. $\mu \mathrm{CT}$ has also been used to show the effects of the PTFE content in the GDL [21], to study the effect of compression on the GDL morphology [22, 23], to identify and segment the MPL within the GDL [24], and to visualize the entire membrane electrode assembly (MEA) [25]. Kim et al. [26] have used $\mu \mathrm{CT}$ to study the porosity variation of paper-based and felt-based GDLs under freeze-thaw cycles and have also provided an extensive review on the evaluation of water management using $\mu \mathrm{CT}$ [27]. These studies show significant value as they can 
be used for numerical models and also for understanding the microstructural transport parameters of the GDL $[22,28]$.

Bazylak and coworkers [6, 29-33] have provided substantial insight into the structure of the GDL by using $\mu \mathrm{CT}$ to acquire a 3D image of the structure of the GDL and then to characterize the porosity distribution. Specifically, Fishman et al. [29-31] have imaged a series of uncompressed GDLs, at a resolution of $2.44 \mu \mathrm{m}$ per voxel side to strategically and sequentially determine the porosity distribution of different types of single layer GDLs (paper, felt, cloth) [29] with different PTFE loadings [30], as well as crack formation in the MPL of dual layer GDLs [31]. The results showed that there is a heterogeneous porosity distribution in the through-plane direction, exposing a more porous surface (due to surface roughness) of the GDL as compared to the core porosity. They also observed a W-shaped pattern in the through plane direction of only paper-based GDLs. This heterogeneous porosity distribution of uncompressed and untreated (no PTFE loading) carbon fiber paper was, by the authors, attributed to the local clustering of the resin binder material in the areas of low porosity formed from a ply layering manufacturing process $[29,7]$. It was found that when the GDL is loaded with PTFE, the porosity decreases mostly in these localized porosity minima, due to the further and preferential agglomeration of PTFE at the same location of the binder material [30]. Furthermore, Fishman et al. studied cracking within the MPL, as well as the interpenetration of the MPL into the carbon fiber paper [31]. In the continuation of this work, Challa et al. $[32,33]$ performed water injection experiments while using $\mu \mathrm{CT}$ to determine the quantitative water profile throughout the GDL at the time of liquid water breakthrough [32]. Additional work compared compressed GDLs to the previous results of the uncompressed GDLs [33]. These comprehensive studies performed by Bazylak and coworkers have provided great insight into the 3D structure and heterogeneous porosity distribution of GDLs. 
The present study conducts a thorough examination of the physical structure of untreated and treated, single and dual layer paper-based GDLs using high-resolution X-ray computed tomographic microscopy. Advanced image analysis methods are presented to segment the different GDL sub-materials from void spaces in order to determine the porosity. The heterogeneous porosity distributions in the through-plane (TP) and in-plane (IP) directions of single layer GDLs are reported. As a novel contribution to previous research, this study also analyses the influence of the MPL's areal weight loading and PTFE content on the intrusion of the MPL into the carbon fiber substrate for dual layer GDLs. Overall, this study reveals the microstructural properties resulting from the various manufacturing processes of single and dual layer GDLs.

\section{Methodology}

This section describes the GDL samples and the procedures that were performed to characterize them.

\subsection{Materials}

In total, eight GDL samples, provided by Ballard Power Systems, were imaged using 3D X-ray computed tomographic microscopy. To investigate the effects of PTFE treatment on the porosity distribution of the GDL, tomographic imaging was performed on five single layer paperbased GDLs from different manufacturers and with varying PTFE ratios. The manufacturers studied were Toray and AvCarb. Specifically, the TGP-H-060 series from Toray and the EP40 series from AvCarb were used for comparison. The PTFE ratio is the proportion of PTFE in the carbon fiber paper by weight (i.e., PTFE/(PTFE+carbon fiber+binder)), with 'T0', 'T6', 'T12', and 'T19' representing $0,6,12$, and $19 \mathrm{wt} \%$, respectively. One main difference between the unteflonated Toray and AvCarb carbon fiber papers (denoted s T0) is that there is an addition of carbon particles in the resin binder of the paper for the AvCarb series [7, 8]. These single layer 
GDL samples will be referred to as TGP-H-060 T0, TGP-H-060 T6, TGP-H-060 T19, EP40 T0 and EP40 T12 throughout the text.

To investigate the effect of the microporous layer (MPL), further $\mu \mathrm{CT}$ scans were conducted on three dual layer GDLs consisting of different PTFE ratios and areal weight loadings. The PTFE ratio, in this case, is the proportion of PTFE in the MPL by weight (i.e., $\mathrm{PTFE} /(\mathrm{PTFE}+$ carbon black)), with 'T18' and 'T30' representing 18 and $30 \mathrm{wt} \%$, respectively. The areal weight loading is the total MPL loading (i.e., PTFE+carbon black), with 'Low' representing $15+/-2 \mathrm{~g} / \mathrm{m}^{2}$ and 'High' representing $35+/-4 \mathrm{~g} / \mathrm{m}^{2}$. The AvCarb EP40 T12 carbon fiber paper is used as the macroporous base substrate upon which the MPL is loaded. In essence, carbon black (CB) from Denka and a PTFE suspension from DuPont were mixed together with a pore forming material (methylcellulose) and deionized water to form the MPL slurry. The slurry was knifecoated onto the carbon fiber paper and the MPL areal weight loading was adjusted by controlling the gap of the blade during the knife coating. The GDL was dried in an oven at $80^{\circ} \mathrm{C}$, then heat treated at $400^{\circ} \mathrm{C}$ for 10 minutes. These dual layer GDL samples will be referred to as EP40 T12 CBT18(Low), EP40 T12 CBT30(Low), and EP40 T12 CBT30(High) throughout the text.

\subsection{Scanning procedure}

The tomographic imaging was performed using an Xradia MicroXCT-400 X-ray computed tomography microscope. The GDL samples were cut to a size of $2 \times 1.5 \mathrm{~mm}$ in cross-section in order to be firmly secured to the sample holder and thus to minimize sample flutter during image acquisition. Approximately $1 \mathrm{~mm}$ x $1 \mathrm{~mm}$ sample size was imaged resulting in volume consisting of approximately $980 \times 980 \times 200$ voxels with a resolution of $1.167 \mu \mathrm{m}$ per voxel side. This satisfies the minimum sample size requirement for repeatable porosity measurement previously prescribed 
by Fishman et al. [29], on the basis of a sensitivity analysis. For each scan, 2500 radiographs, scanning $360^{\circ}$, were taken at an exposure time of $10 \mathrm{~s}$.

\subsection{Image processing}

For all scans, post-processing was performed using ImageJ and Avizo to segment the GDL material from void space (representing pores). As the binder/PTFE and carbon fiber strands have a similar grey-scale value, it was not possible to differentiate between them; hence they will collectively be referred to as fibers. Also, due to the resolution limit of the Xradia tomography apparatus of $\sim 1 \mu \mathrm{m}$, porosity within the MPL cannot be identified and it is treated as a bulk material $^{1}$. Although X-ray scattering effects can sometimes be a problem in micro-CT imaging resulting in difficulties in edge detection [37], the issue was not observed in the series of scans performed for this work. After imaging was complete, all the scans were rotated to align the $x-y-$ z- axes of the GDL to the global coordinate system, and then cropped to the area of interest. A through-plane (TP) view refers to a cross-section parallel to the thickness direction while an inplane (IP) view refers to a cross-section parallel to the plane of the GDL sheet.

\subsubsection{Single layer GDLs}

Single layer GDLs were taken through the following image processing sequence. First, a 3D anisotropic diffusion filter [38] was applied to the 3D dataset in order to reduce noise. The anisotropic diffusion filter uses the gradient information in the image to define a function that smoothes the image along the edges while preserving the information across them. This results in enhanced edges separating homogeneous regions which facilitates the selection of a hard threshold

\footnotetext{
${ }^{1}$ The nanometer scale porosity of MPL can be resolved using other methods, such as nano-CT methods or destructive microscopy, i.e. focused ion-beam scanning-electron microscopy such as used by Gunda et al. [36]. Nevertheless, the lack of resolution for imaging porosity within the MPL does not affect characterization of the intrusion of MPL within the carbon fiber substrate of the dual-layer GDL.
} 
for segmentation of fibers from air. Second, a global threshold was manually selected to isolate the fibers from the background. Ostadi et al. [34] have indicated the importance of selecting proper thresholding values. Finally, a morphological closing operation was applied within the ImageJ software to eliminate small holes arising due to inhomogeneity in the data.

\subsubsection{Dual layer GDLs}

Figure 1(a) shows a TP view (cropped) of dual layer EP40 T12 CBT18(Low) GDL. The MPL, located in the upper half of the image, appears light grey; the GDL fibers (including binder and PTFE) appear white, and the void space is dark grey. As it can be seen, the void space and MPL have similar grey scale values, and thus a more rigorous approach for segmentation was required as compared to the single layer GDLs. Dual layer GDLs were taken through the following image processing sequence, visually outlined in Figure 1. Firstly, two 2D binary datasets were created using the methodology described in 2.3.1. In one dataset, Figure 1(b), a global threshold was applied such that both the MPL and the fibers were segmented from the background. In the other dataset, Figure 1(c), a different global threshold was applied to segment only the fibers while the MPL was included with the background. Secondly, the first dataset was multiplied against the invert of the second, resulting in MPL segmentation as shown in Figure 1(d).

\subsubsection{Quantification}

Once the segmentation was completed, image analysis tools were used on the segmented 2D slices of the GDL in order to determine the area porosity as $\varepsilon=100-\mathrm{A}$, where $\mathrm{A}$ is the area percentage of the GDL material in the image (including the fibers, binder and PTFE, and/or the MPL material). To quantify the porosity distribution within the single layer GDLs, the IP slices were used to determine the TP porosity distribution traversing the GDL thickness and the two TP slices were used to determine two IP porosity distributions traversing along the GDL sheet. Since 
there is experimental noise at the ends of the sample, the IP directional porosity distribution data considered for analysis has been cropped from $-350 \mu \mathrm{m}$ to $+350 \mu \mathrm{m}$, which still includes the entire GDL thickness $(\sim-100 \mu \mathrm{m}$ to $\sim+100 \mu \mathrm{m})$, where the 0 represents the mid-point of the GDL. The bulk porosity is obtained by dividing the number of voxels of void space by the total number of voxels in the stack of images. Only image slices containing at least $1 \%$ GDL were included in the calculation of bulk porosity, following Fishman et al. [30]. For dual layer GDLs, the area percentage of the MPL was quantified as a function of the thickness of the GDL.

\section{Results and Discussion}

This section shows qualitative and quantitative results for the single layer GDLs, as well as the results from the analysis performed on the dual layer GDLs.

\subsection{Analysis of single layer GDLs}

\subsubsection{Qualitative}

An IP slice of AvCarb EP40 T0 GDL, taken from the 3D dataset is shown in Figure 2(a), along with an SEM image (Figure 2(b)) for comparison purposes. As it can be seen, the tomographic image shows all the main features captured in the SEM image, i.e. the carbon strands, resin binder and void space. It would appear that the porosity was created by two types of voids: macropores from bulk voids between the carbon strands, and micropores within the binder filled with carbon particles. Although the GDL structure looks much simpler in the binary cross-sectional slices as compared to the SEM image, the web-like morphology between the binder and carbon fiber strands can be clearly observed in both images.

Figure 3 shows a reconstructed 3D image of TGP-H-060 T19 (Figure 3(a)), along with cross-sectional views from the IP (Figure 3(b)), and TP (Figures 3(c, d)) slice directions. As it can be seen in Figure 3(a), the PTFE visually appears to be distributed throughout the GDL in a non- 
uniform manner; some areas rich with PTFE while others are nearly transparent. The carbon fiber strands, while mainly randomly distributed and oriented, may contain slight machine directionality from the manufacturing process. Figure 3(b) displays the binary IP slice, in which the long, thin white areas represent the carbon strands, and the larger white areas correspond to a local area of PTFE webbing between the carbon strands. The TP slices (Figures 3(c, d) show that the surface of the single layer GDL has inherent surface roughness, which is known to increase contact resistance. These figures also reveal both through pores, allowing reactants and products to flow through the GDL, and closed or dead end pores.

\subsubsection{Quantitative}

The bulk porosity and standard deviation of the area porosity (as compared to the bulk value) from $\mu \mathrm{CT}$ measurements, as well as the average pore diameters (determined via MSP [12]), are presented in Table 1 for both unteflonated and teflonated single layer Toray TGP-H-060 series and AvCarb EP40 series GDLs. As can be seen, there is considerable variation in porosity between the different series of single layer GDLs and within a GDL series as a function of PTFE content. Note that each value of porosity is from a single tomography scan, and there could be variations within a GDL sheet or between different GDL sheets of the same material. Also, although the segmentation was robust, changes in the chosen threshold value for the segmentation would also change the reported bulk porosity.

The results given in Table 1 are plotted in Figure 4, showing the variation in bulk porosity as a function of the PTFE content for both the Toray and AvCarb GDLs, along with prior data from Fishman et al. [30] for TGP-H-060 GDLs. Although the porosity results in this work (for the Toray TGP-H-060 series) follow the same trend as prior findings, the actual magnitudes are much different. This could be due to either differences in the manufacturing process, density variations 
within the GDL sheet, and differences in the method used to denoise and binarize the data. While the addition of PTFE had a significant effect on the porosity in the Toray series GDLs (with a decrease from $82 \%$ to $78 \%$ to $71 \%$ for TGP-H-060 T0, T6, and T19, respectively), no significant change in the porosity was found for the AvCarb series GDLs upon addition of PTFE ( $82 \%$ for both EP40 T0 and T12). This result was unexpected, but as noted in [30], the nature of GDL manufacturing is batch-specific and there could be significant variation in percentage porosity between GDL sheets. This porosity variability, as well as the porosity variability on any individual GDL sheet will be the focus of a future study.

The main benefit of $\mu \mathrm{CT}$ is the ability to characterize property variation along any direction vector within the GDL. Figure 5 shows the variation in porosity in the TP direction for all five single layer GDLs. As it can be seen, there is a considerable variation in the area porosity as a function of position. For example, the TP heterogeneous area porosity of the unteflonated TGP-H060 T0 GDL has a W-shape, with a sharp drop in porosity near both surfaces (due to surface roughness) and a core region with peaks and valleys of local maxima and minima. Also, the distribution is not symmetric; with a lower minimum on one side of the GDL as compared to the other. As discussed earlier, Fishman et al. [29] have attributed this characteristic W-shaped pattern to the ply manufacturing process that is used in the production of paper-based GDLs and the agglomeration of binder in local minima porosity regions $[29,7]$. The TP direction porosity for the teflonated samples is affected most in the minima regions of porosity found in the unteflonated sample, due to the further and preferential agglomeration of the PTFE in the local minima regions [30]. All of the five curves show the same trend. However, the variations are enhanced in the Toray series GDLs as compared to the AvCarb series GDLs since, as shown in Figure 4, the bulk porosity of the Toray material decreases significantly with increasing PTFE content, while the decrease in 
porosity for the AvCarb material is limited. This difference could be due to production methods, since the Toray GDL is manufactured using a ply process, whereas the AvCarb GDL is manufactured via bulk consolidation. Thus the limited W-shaped pattern seen in the AvCarb GDL could simply be due to material heterogeneity.

The porosity distribution in both the two IP directions and the TP direction for Toray TGPH-060 T19 is shown in Figure 6. As it can be seen, the area percentage porosity in both IP directions fluctuates marginally and randomly, and matches well with the bulk porosity value, whereas there is great variation in the TP direction porosity. These results follow the trends that have been reported in other studies using $\mu \mathrm{CT}[29,30]$, and may be in part from the preferred gathering of PTFE in the lower porosity regions of the untreated GDL, and/or in part from the drying process of the PTFE in the GDL $[29,30,7,21]$.

The results displayed in Table 1 show a much different influence of the effect of PTFE on the average pore diameter as compared to the bulk porosity differences between the Toray and AvCarb series GDLs. While the expected trend of a decrease in pore size with an increase in PTFE loading was found, the average pore size for the Toray only decreased slightly (reducing from 33 to 30.5 to $29 \mu \mathrm{m}$ for TGP-H-060 T0, T6, and T19, respectivley) in comparison to AvCarb GDLs (52 to $27.5 \mu \mathrm{m}$ for EP40 T0 and T12, respectively). Han et al. [8] has found similar trends between single layer and carbon-filled GDLs (CFGDLs).

The results given in Figures 4-6 and in Table 1 indicate that there is a complex relationship between the interconnected voids creating the macropores and micropores present in the GDL. It can be hypothesized that these microstructural changes in the GDL are caused by (i) the manufacturing process and more specifically the application and drying methods of PTFE and (ii) the intrinsic manner in which the PTFE interacts with the base carbon fibers used to make the Toray 
and AvCarb series GDLs. It should be mentioned that the results for bulk porosities and trends for the effect of PTFE treatment match well with other methods of porosimetry reported in literature $[8,9]$. As shown in this section, PTFE can locally reduce porosity, and hence alter transport properties of the GDL. Using the results from $\mu \mathrm{CT}$, a better understanding of porosity distributions in current GDL manufacturing processes can be obtained, in an effort to optimize the underlying parameters for improved fuel cell performance.

\subsection{Analysis of dual layer GDLs}

A quantitative comparison has been conducted to study the effect of the addition of a microporous layer (MPL) to a macroporous paper substrate. Figure 7(a) presents the dual layer EP40 T12 CBT18(Low) GDL, while Figure 7(b) presents the single layer EP40 T12 GDL. Note that the greyscale value of the pixels corresponding to MPL in Figure 7(a) have been modified so that the MPL (light grey) can be easily distinguished from the void space (dark grey) and the GDL fibers (including binder/PTFE) (white). The comparison of the two images shows that the MPL percolates deeply into the carbon fiber paper in specific regions, and reveals a variation of the intrusion depth of the MPL into the carbon paper. The dual layer GDL (Figure 7(a)) is thicker as compared to the single layer GDL (Figure 7(b)) due to the addition of MPL material. The presence of the MPL has also decreased the surface roughness of the GDL, from 26 microns to 5 microns. Hence, the contact resistance between the GDL and catalyst layer will be decreased. Note that the surface roughness was quantified by calculating the root mean squared variation of the values of the distances of every point on the surface from the mean surface profile [39] in both Figure 7(a) and (b).

While some have reported a clear boundary between the MPL on the macroporous substrate [8], many others have reported a complex interaction between the MPL material intruding into the 
carbon fiber paper $[8,9,13,24,31]$, corroborating the results shown in Figure 7 . Fishman et al. [31] has effectively shown the effect of cracking within the MPL using $\mu$ CT. In a similar fashion, Pfrang et al. [24] separated the MPL and fibers (including PTFE) to determine their respective filled fraction contributions as a function of the GDL thickness. The reasons behind the cracking and intrusion of the MPL is highly dependent on the material recipe, manufacturing process, drying procedure and heat treatment used for the fabrication and application of the MPL onto the carbon fiber paper.

Through advanced image analysis, the MPL was segmented from the carbon fiber paper (including binder and PTFE), and the area percentage of the MPL was quantified as a function of the GDL thickness. Figure 8 compares the area percentage of the MPL in the TP direction with the three dual layer GDLs. The zero on the X-axis represents the side of the carbon paper that the MPL was not applied to. The results from MPLs with different PTFE levels and the same areal weight loadings (EP40 T12 CBT18(Low) and CBT30(Low)) demonstrate that with a lower PTFE level (CBT18(Low)), there is more intrusion of the MPL into the carbon paper in comparison to a higher PTFE level (CBT30(Low)). The researchers hypothesize that this trend may be caused by the difference in the viscosity of the MPL slurry, since a previous study indicates that an increase of PTFE content in the MPL slurry results in an increase in viscosity [40]. Based on this hypothesis, the greater viscosity in the CBT30(Low and High) MPLs, as compared to the CBT18(Low) MPL, would inhibit the MPL slurry from infiltrating the GDL material during application.

The results from MPLs with the same PTFE level and different areal weight loadings (EP40 T12 CBT30(Low) and CBT30(High)) show that with a higher areal weight loading (CBT30(High)), the MPL becomes almost twice as thick in comparison to a lower areal weight loading (CBT30(Low)). There is a region near the CBT30(High) MPL surface where the area 
percentage reaches nearly $100 \%$ due to a dense agglomeration of MPL material from the higher areal weight loading. Figure 8 shows that, for these more viscous MPLs (with higher PTFE levels), there is a greater accumulation of the MPL towards the side of the carbon paper that the MPL was applied to, with less interpenetration into the bulk of the carbon paper. Even when more material is added with the CBT30(High) MPL, there is not as much invasion as compared with the CBT18(Low) MPL. This leads to the conclusion that the PTFE level in the MPL will restrict the intrusion of the MPL into the carbon fiber paper. Thus, by using $\mu \mathrm{CT}$, valuable information about the interaction of the MPL with the carbon fiber paper was inferred, which can lead to advanced understanding of this crucial layer for enhanced fuel cell performance.

\section{Conclusions}

This study utilized high-resolution X-ray computed tomographic microscopy to acquire 3D images of Toray TGP-H-060 and AvCarb EP40 series carbon paper GDLs. Through analysis it was found that the variation in bulk porosity and average pore diameter of single layer GDLs depends highly on the GDL series manufacturing and treatment processes. In general, the heterogeneous (W-shape) porosity distribution found in the TP direction of the paper-based GDLs is attributed to the ply manufacturing process, the preferential agglomeration of binder and PTFE, and the procedure used for the drying process of the PTFE. Furthermore, dual layer GDLs were examined in order to evaluate the interaction between the MPL and the carbon fiber paper. Using advanced image analysis techniques, routines were developed to accurately segment the GDL fibers (including binder/PTFE) and the MPL. The percentage of the intruding MPL material into the carbon fiber paper as a function of GDL thickness was determined for dual layer GDLs that have varying PTFE content and areal weight loading in the MPL. This analysis provides invaluable insight into the physical microstructure of paper-based GDLs, emphasizing the heterogeneous 
porosity distribution of single layer GDLs and the interaction of the MPL with the carbon fiber paper of dual layer GDLs.

\section{Acknowledgements}

The authors wish to thank The Natural Sciences and Engineering Research Council of Canada (NSERC) and the Canada Foundation for Innovation (CFI) for funding this research. The support of The University of British Columbia in the form of an International Undergraduate Research Award is also gratefully acknowledged. 


\section{References}

[1] Stambouli, A., \& Traversa, E. (2002). Fuel cells, an alternative to standard sources of energy. Renewable and Sustainable Energy Reviews, 6(3), 297-306.

[2] Cindrella, L., Kannan, A. M., Lin, J. F., Saminathan, K., Ho, Y., Lin, C. W., et al. (2009). Gas diffusion layer for proton exchange membrane fuel cells-A review. Journal of Power Sources, 194(1), 146-160.

[3] Arvay, A., Yli-Rantala, E., Liu, C., Peng, X., Koski, P., Cindrella, L., et al. (2012). Characterization techniques for gas diffusion layers for proton exchange membrane fuel cells - A review. Journal of Power Sources, 213, 317-337.

[4] Park, S., Lee, J., \& Popov, B. N. (2012). A review of gas diffusion layer in PEM fuel cells: Materials and designs. International Journal of Hydrogen Energy, 37(7), 5850-5865.

[5] Jordan, L. R., Shukla, A. K., Behrsing, T., Avery, N. R., Muddle, B. C., \& Forsyth, M. (2000). Diffusion layer parameters influencing optimal fuel cell performance. Journal of Power Sources, 86(1), 250-254.

[6] Bazylak, A. (2009). Liquid water visualization in PEM fuel cells: A review. International Journal of Hydrogen Energy, 34(9), 3845-3857.

[7] Mathias, M., Roth, J., Fleming, J., and Lehnert, W. L. (2003). Diffusion media materials and characterization. In W. Vielstich, H. A. Gasteiger, A. Lamm (Eds.), Handbook of Fuel Cells: Fundamentals, Technology and Applications (Vol. 3, pp. 1-21). New York, NY: Wiley \& Sons.

[8] Han, M., Xu, J. H., Chan, S. H., \& Jiang, S. P. (2008). Characterization of gas diffusion layers for PEMFC. Electrochimica Acta, 53(16), 5361-5367.

[9] Martinez-Rodriguez, M., Tong, C., Shimpalee, S., \& Van Zee, J. W. (2010). Characterization of microporous layer in carbon paper GDL for PEM fuel cell. 10th Polymer Electrolyte Fuel Cell Symposium, PEFC 10 - 218th ECS Meeting, October 10, 2010 - October 15, 33. (1) pp. 1133-1141.

[10] Park, G., Sohn, Y., Yang, T., Yoon, Y., Lee, W., \& Kim, C. (2004). Effect of PTFE contents in the gas diffusion media on the performance of PEMFC. Journal of Power Sources, 131(1-2), 182-187.

[11] Parikh, N., Allen, J. S., \& Yassar, R. S. (2012). Microstructure of gas diffusion layers for PEM fuel cells. Fuel Cells, 12(3), 382-390.

[12] Phillips, R. K., Friess, B. R., Hicks, A. D., Bellerive, J., \& Hoorfar, M. (2012). Ex-situ measurement of properties of gas diffusion layers of PEM fuel cells. 19th World Hydrogen Energy Conference, WHEC 2012, June 3, 2012 - June 7, 29. pp. 486-495.

[13] Gostick, J. T., Fowler, M. W., Ioannidis, M. A., Pritzker, M. D., Volfkovich, Y. M., \& Sakars, A. (2006). Capillary pressure and hydrophilic porosity in gas diffusion layers for polymer electrolyte fuel cells. Journal of Power Sources, 156(2), 375-387.

[14] Sinha, P. K., Halleck, P., \& Wang, C. (2006). Quantification of liquid water saturation in a PEM fuel cell diffusion medium using X-ray microtomography. Electrochemical and Solid-State Letters, 9(7), A344-A348. 
[15] Buchi, F. N., Fluckiger, R., Tehlar, D., Marone, F., \& Stampanoni, M. (2008). Determination of liquid water distribution in porous transport layers. Proton Exchange Membrane Fuel Cells 8, PEMFC - 214th ECS Meeting, October 12, 2008 - October 17, 16. (2) pp. 587592.

[16] Fluckiger, R., Marone, F., Stampanoni, M., Wokaun, A., \& Buchi, F. N. (2011). Investigation of liquid water in gas diffusion layers of polymer electrolyte fuel cells using X-ray tomographic microscopy. Electrochimica Acta, 56(5), 2254-2262.

[17] Suzuma, I., Ejiri, E., Hirono, M., Takimoto, M., \& Yoneda, M. (2009). Measurements of water droplet behavior in gas diffusion layer of PEFC using X-ray CT and substance microscope. Student Posters - General - 214th ECS Meeting/PRiME 2008, October 12, 2008 - October 17, 16. (24) pp. 133-140.

[18] Gostick, J. T., Gunterman, H. P., Kienitz, B. W., Newman, J. S., MacDowell, A. A., \& Weber, A. Z. (2010). Tomographic imaging of water injection and withdrawal in PEMFC gas diffusion layers. 10th Polymer Electrolyte Fuel Cell Symposium, PEFC 10 - 218th ECS Meeting, October 10, 2010 - October 15, 33. (1) pp. 1407-1412.

[19] Markotter, H., Manke, I., Kruger, P., Arlt, T., Haussmann, J., Klages, M., et al. (2011). Investigation of $3 \mathrm{D}$ water transport paths in gas diffusion layers by combined in-situ synchrotron X-ray radiography and tomography. Electrochemistry Communications, 13(9), 1001-1004.

[20] Markotter, H., Haumann, J., Alink, R., Dittmann, K., Totzke, C., Kruger, P., et al. (2013). Investigation of fuel cell materials and liquid water transport by means of synchrotron imaging. Symposium on Batteries and Energy Technology Joint General Session - 221st ECS Meeting, may 6, 2012 - May 10, 45. (29) pp. 195-202.

[21] Sasabe, T., Inoue, G., Tsushima, S., Hirai, S., Tokumasu, T., \& Pasaogullari, U. (2012). Investigation on effect of PTFE treatment on GDL micro-structure by high-resolution Xray CT. 12th Polymer Electrolyte Fuel Cell Symposium, PEFC 2012 - 222nd ECS Meeting, October 7, 2012 - October 12, , 50. (2) pp. 735-744.

[22] Je, J., Doh, S., Kim, J., \& Kim, M. H. (2012). Heterogeneous porosity distribution under compression condition of gas diffusion layer using synchrotron X-ray tomography. 12th Polymer Electrolyte Fuel Cell Symposium, PEFC 2012 - 222nd ECS Meeting, October 7, 2012 - October 12, 50. (2) pp. 369-374.

[23] Diedrichs, A., Rastedt, M., Pinar, F. J., \& Wagner, P. (2013). Effect of compression on the performance of a HT-PEM fuel cell. , 43. (11) pp. 1079-1099.

[24] Pfrang, A., Didas, S., \& Tsotridis, G. (2013). X-ray computed tomography of gas diffusion layers of PEM fuel cells: Segmentation of the microporous layer. Journal of Power Sources, 235, 81-86.

[25] Pfrang, A., Veyret, D., Janssen, G. J. M., \& Tsotridis, G. (2011). Imaging of membrane electrode assemblies of proton exchange membrane fuel cells by $\mathrm{X}$-ray computed tomography. Journal of Power Sources, 196(12), 5272-5276.

[26] Kim, S., \& Lee, S. (2012). Tomographic analysis of porosity variation in gas diffusion layer under freeze-thaw cycles. International Journal of Hydrogen Energy, 37(1), 566-574. 
[27] Kim, S., \& Lee, S. (2013). A review on experimental evaluation of water management in a polymer electrolyte fuel cell using X-ray imaging technique. Journal of Power Sources, 230, 101-108.

[28] Becker, J., Fluckiger, R., Reum, M., Buchi, F. N., Marone, F., \& Stampanoni, M. (2009). Determination of material properties of gas diffusion layers: Experiments and simulations using phase contrast tomographic microscopy. Journal of the Electrochemical Society, 156(10), B1175-B1181.

[29] Fishman, Z., Hinebaugh, J., \& Bazylak, A. (2010). Microscale tomography investigations of heterogeneous porosity distributions of PEMFC GDLs. Journal of the Electrochemical Society, 157(11), B1643-B1650.

[30] Fishman, Z., \& Bazylak, A. (2011). Heterogeneous through-plane porosity distributions for treated PEMFC GDLs I. PTFE effect. Journal of the Electrochemical Society, 158(8), B841-B845.

[31] Fishman, Z., \& Bazylak, A. (2011). Heterogeneous through-plane porosity distributions for treated PEMFC GDLs. II. Effect of MPL cracks. Journal of the Electrochemical Society, 158(8), B846-B851.

[32] Challa, P., Hinebaugh, J., \& Bazylak, A. (2011). Comparison of water thickness profiles of compressed PEMFC GDLS. Collocated with ASME 2011 5th International Conference on Energy Sustainability, FUELCELL 2011, August 7, 2011 - August 10, pp. 121-126.

[33] Challa, P. R., \& Bazylak, A. (2012). Pore space characterization of compressed PEMFC GDLS using 3D microcomputed tomography. ASME 2012 10th International Conference on Fuel Cell Science, Engineering and Technology, FUELCELL 2012 Collocated with the ASME 2012 6th International Conference on Energy Sustainability, July 23, 2012 - July 26, pp. 189-194.

[34] Ostadi, H., Rama, P., Liu, Y., Chen, R., Zhang, X. X., \& Jiang, K. (2010). Influence of threshold variation on determining the properties of a polymer electrolyte fuel cell gas diffusion layer in X-ray nano-tomography. Chemical Engineering Science, 65(6), 22132217.

[35] Maurer, C.R., Qi, R., and Raghavan, V. (2003). A linear time algorithm for computing exact euclidean distance transforms of binary images in arbitrary dimensions. IEEE Trans. Pattern Anal. Mach. Intell., 25 (2): 265-270.

[36] Gunda, N.S., Choi, H.W., Berson, A., Kenney, B., Karan, K., Pharoah, J.G., \& Mitra, S.K. (2011). Focused ion beam-scanning electron microscopy on solid-oxide fuel-cell electrode: Image analysis and computing effective transport properties. Journal of Power Sources, 196, 3592-3603.

[37] Bera, B., Mitra, S.K., \& Vick, D. (2011). Understanding the micro structure of Berea Sandstone by the simultaneous use of micro-computed tomography (micro-CT) and focused ion beam-scanning electron microscopy (FIB-SEM). Micron, 42, 412-418.

[38] Weickert, J., Romeny, B.M.H., Viergever, M.A. (1998). Efficient and reliable schemes for nonlinear diffusion filtering. IEEE Trans. Image Proc., 7(3): 398-410. 
[39] Degarmo, P.E., Black, J.T., \& Kohser, A.R. (2003). Materials and processes in manufacturing. Wiley, $9^{\text {th }}$ edition, 223.

[40] Furuya, N., \& Mineo, N. (2007). Preparation of a concentrated carbon black-PTFE dispersion for micro-porous layer of PEMFC. Journal of New Materials for Electrochemical Systems, 10, 205-208. 
Table 1: Porosity analysis (bulk porosity, average pore diameter, and standard deviation in area porosity in the three directions) of Toray TGP-H-060 T0, T6, T19 and AvCarb EP40 T0, T12. The Average pore diameter was measured via MSP while the remaining values were acquired from $\mu \mathrm{CT}$ imaging.

\begin{tabular}{lccccc}
\hline GDL Type & $\begin{array}{c}\text { Bulk } \\
\text { Porosity } \\
(\mathbf{\%})\end{array}$ & $\begin{array}{c}\text { Average pore } \\
\text { diameter } \\
(\boldsymbol{\mu m})\end{array}$ & $\begin{array}{c}\text { TP Area } \\
\text { Porosity SD } \\
(\mathbf{\% )}\end{array}$ & $\begin{array}{c}\text { IP-1 Area } \\
\text { Porosity SD } \\
(\boldsymbol{\%})\end{array}$ & $\begin{array}{c}\text { IP-2 Area } \\
\text { Porosity SD } \\
\mathbf{( \% )}\end{array}$ \\
\hline TGP-H-060 T0 & 82.3 & 32.92 & 8.9 & 1.3 & 2.5 \\
TGP-H-060 T6 & 78.3 & 30.48 & 12.8 & 1.6 & 1.2 \\
TGP-H-060 T19 & 71.1 & 29.03 & 12.5 & 2.0 & 2.9 \\
EP40 T0 & 82.4 & 52.00 & 8.5 & 3.0 & 2.4 \\
EP40 T12 & 82.2 & 27.53 & 10.2 & 2.9 & 2.6 \\
\hline
\end{tabular}




\section{Figure Captions}

Figure 1: TP section of EP40 T12 CBT18 (Low) taken from the 3D tomographic dataset; (a) the raw grey scale image showing the contrast between the GDL fibers (incl. binder/PTFE) (white), the MPL (light grey) and void space (dark grey); (b) the binary image of MPL and GDL (white), and void space (black); (c) the binary image of the void space and MPL (black), and the GDL fibers (white); (d) the final binarized view of the MPL (black) used for image analysis

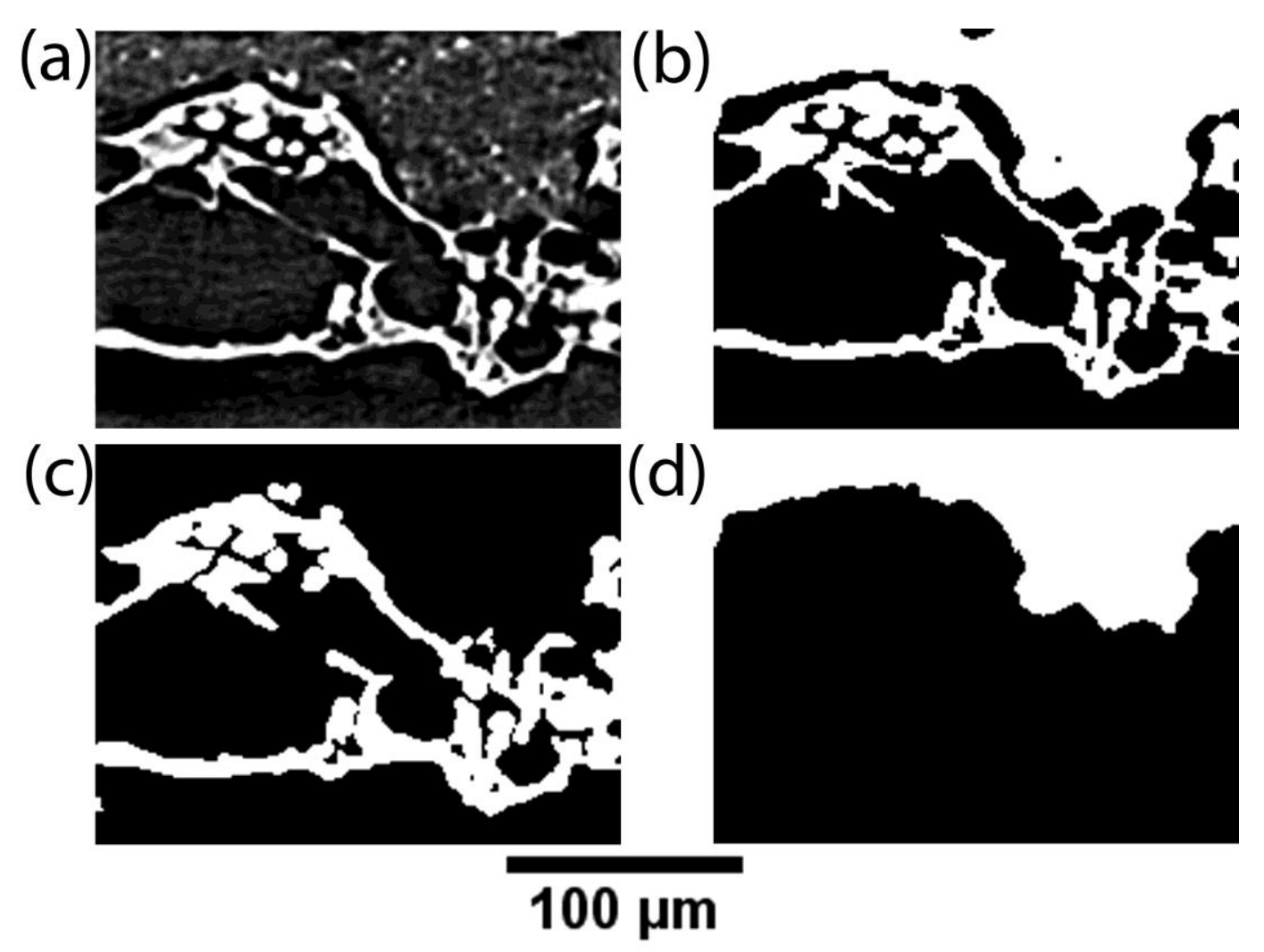


Figure 2: Comparison of (a) cross-sectional view from the 3D tomographic dataset of AvCarb EP40 T0 and (b) SEM image.

a)
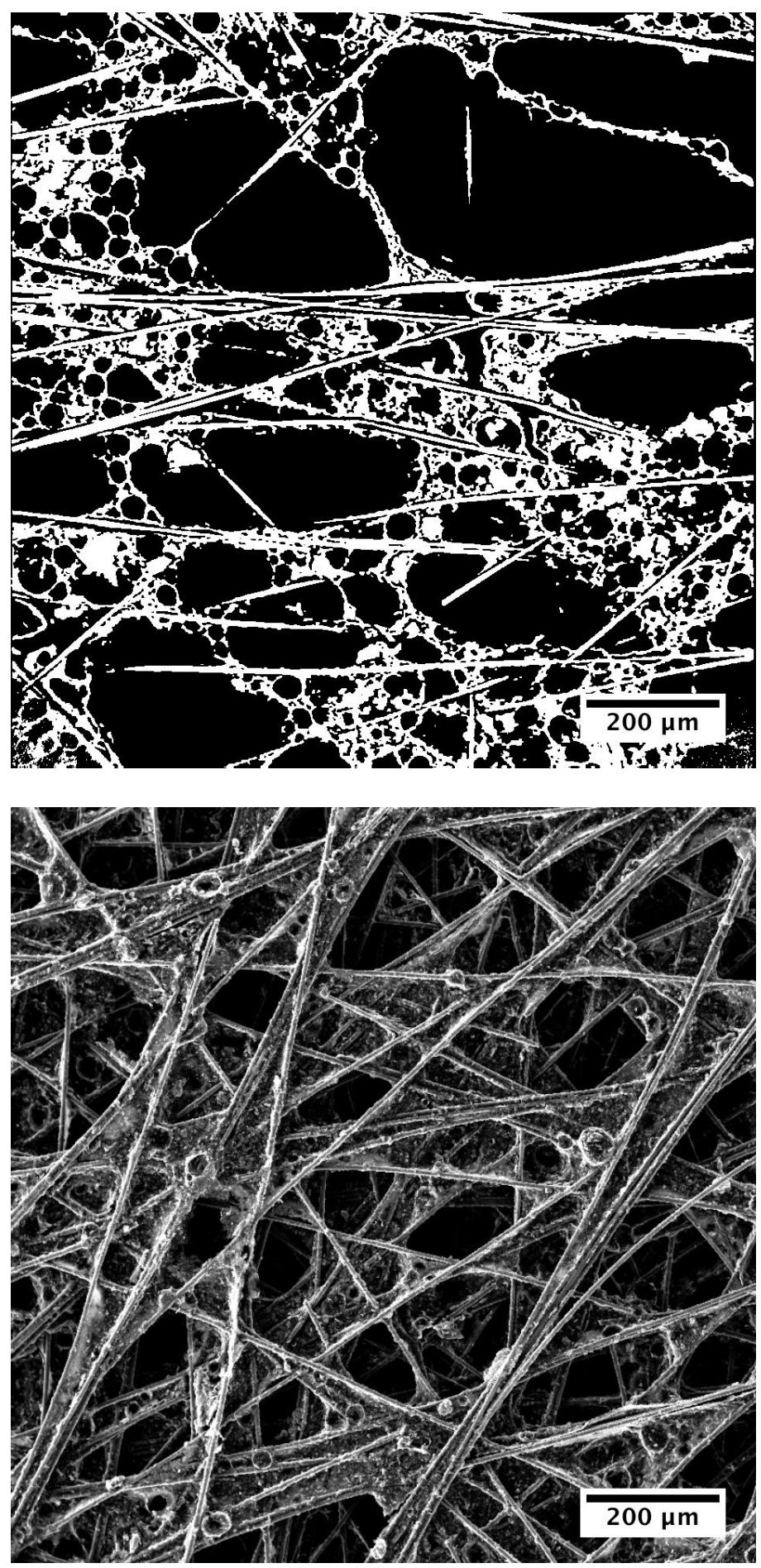
Figure 3: Tomographic image of Toray TGP-H-060 T19; (a) 3D volume rendering of the carbon fibers, binder and PTFE; (b)-(d) cross-sectional slices in the IP, TP-1, and TP-2 directions, respectively

a)

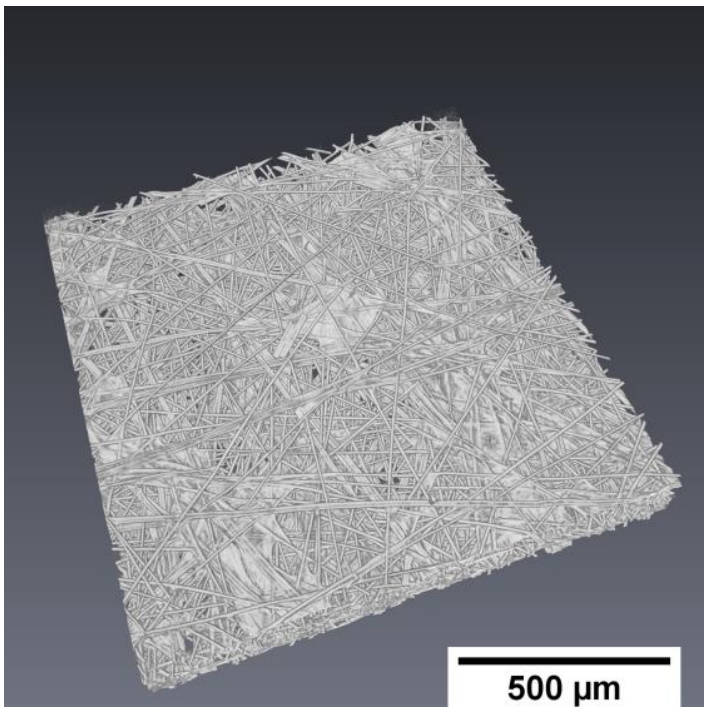

b)

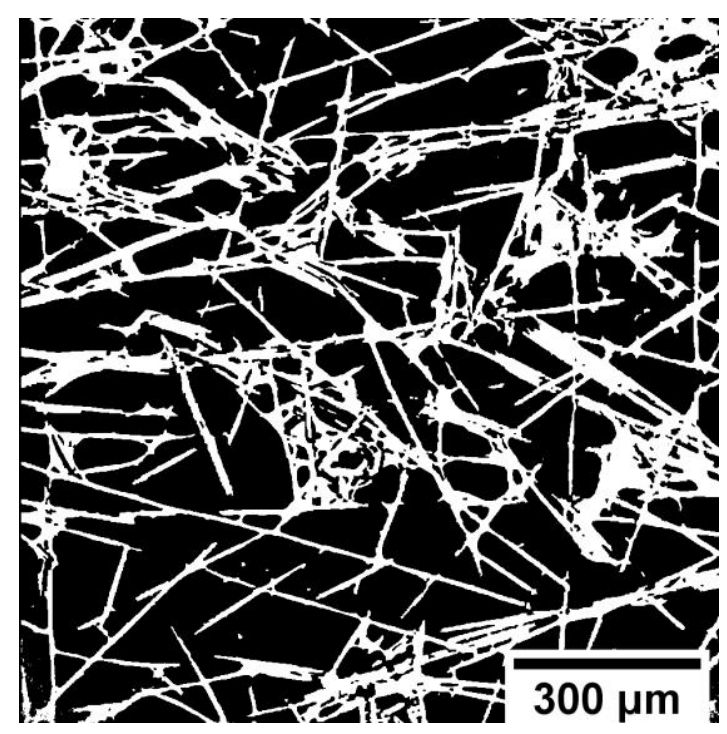

c)

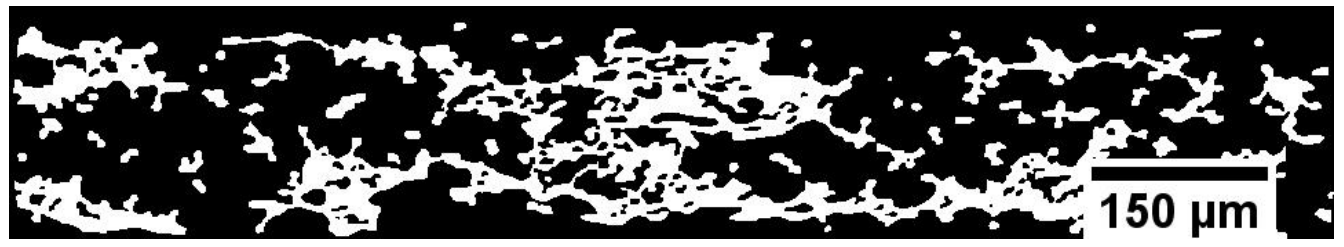

d)

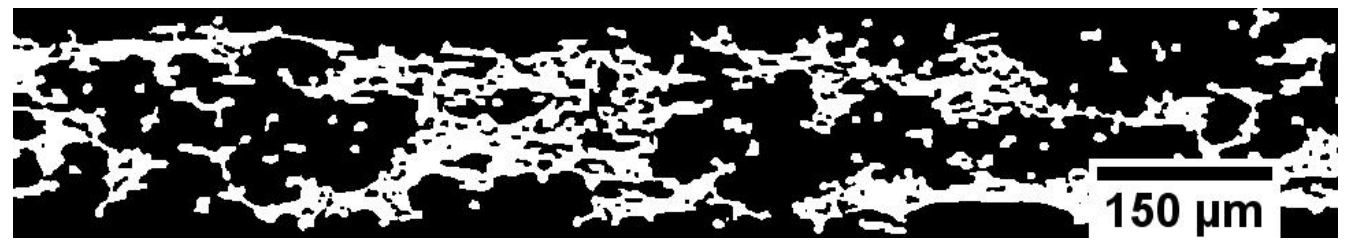


Figure 4: Variation in bulk porosity with PTFE content for Toray TGP-H-060 and AvCarb EP40 GDL samples. Note: Literature data [Fishman-i(2011)] for Toray TGP-H-060 series is provided for comparison purposes.

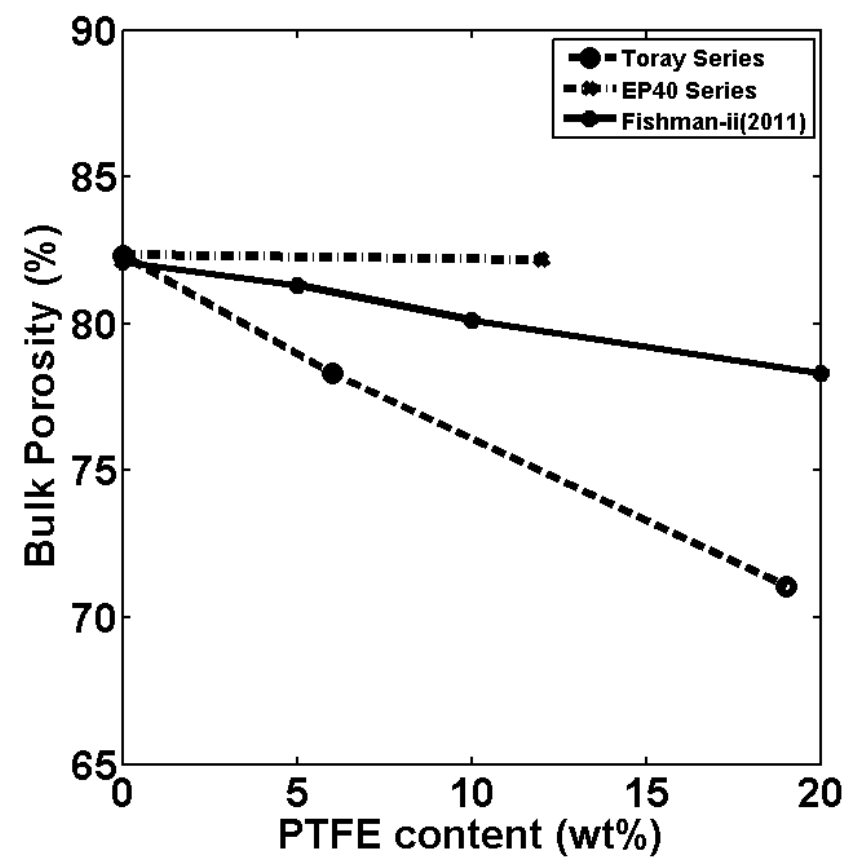

Figure 5: Porosity distributions in the TP direction for different amounts of PTFE treatment (Toray TGP-H-060 T0, T6, and T19 and AvCarb EP40 T0 and T12)

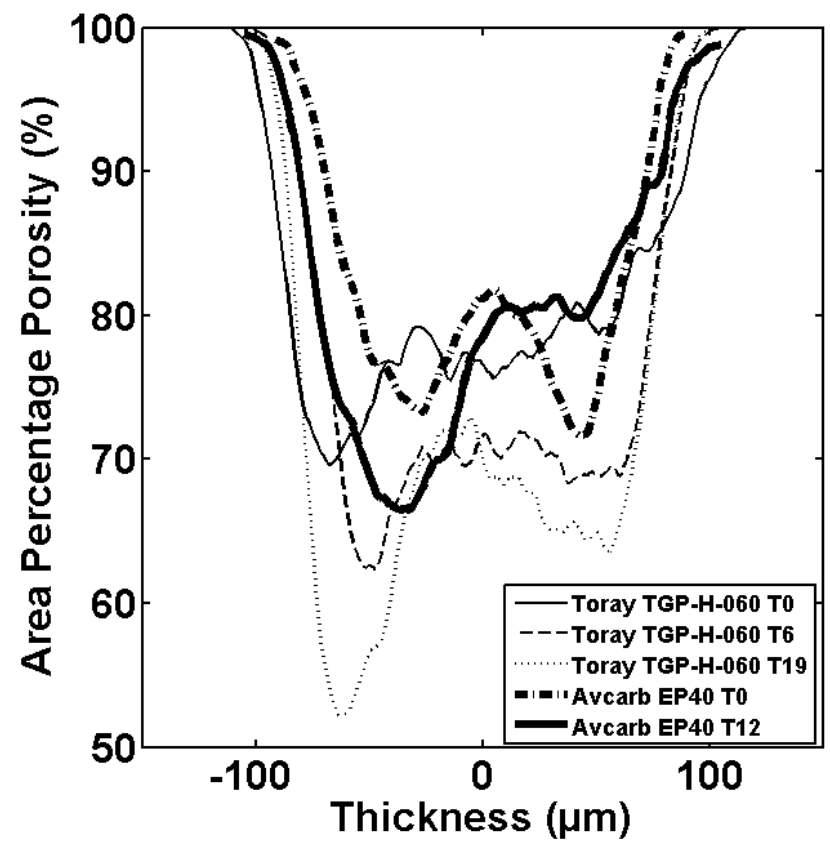


Figure 6: Porosity distribution in the TP direction and IP directions for Toray TGP-H-060 T19

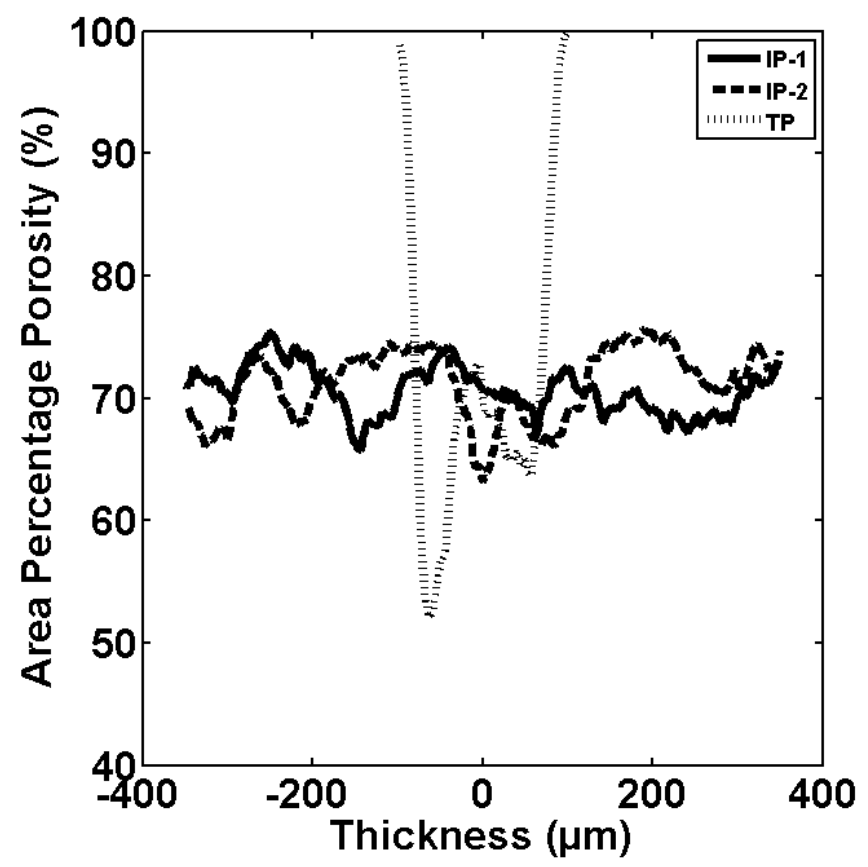

Figure 7: Tomographic image of a cross-sectional slice of (a) dual layer GDL EP40 T12 CBT18 (Low); (b) single layer GDL EP40 T12

a)

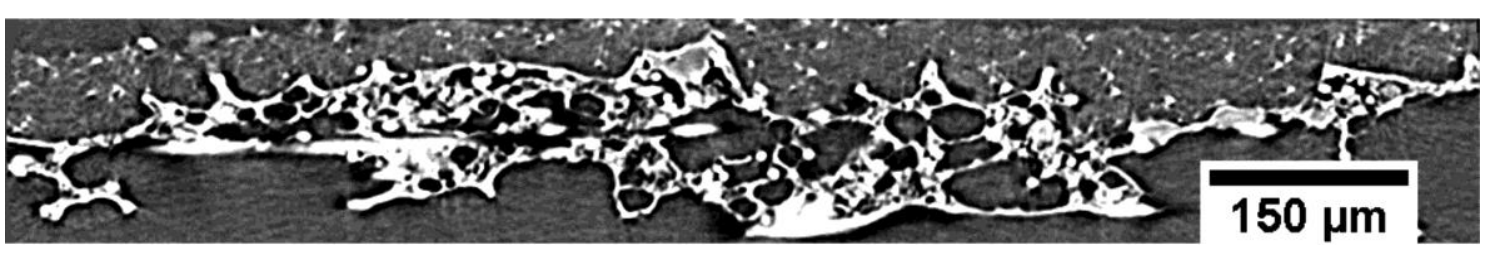

b)

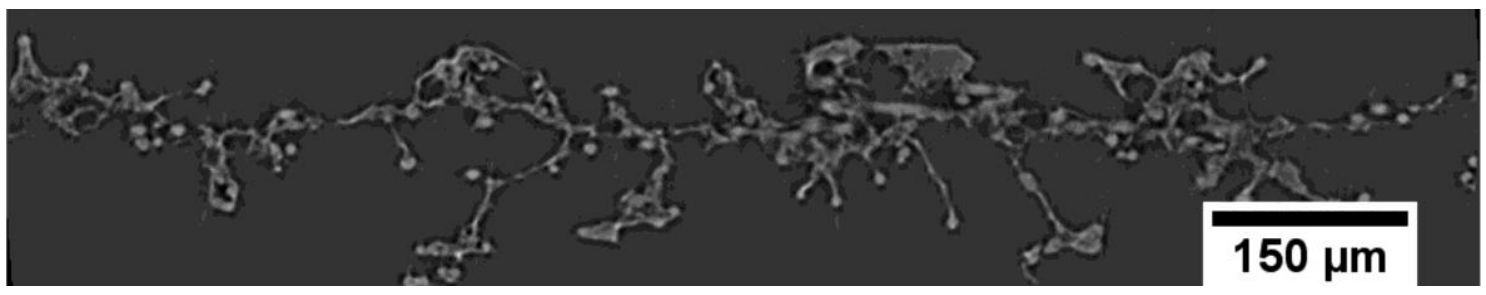


Figure 8: Area percentage distribution of MPL with dual layer GDLs; EP40 T12 CBT18 (Low), EP40 T12 CBT30 (Low), EP40 T12 CBT30 (High)

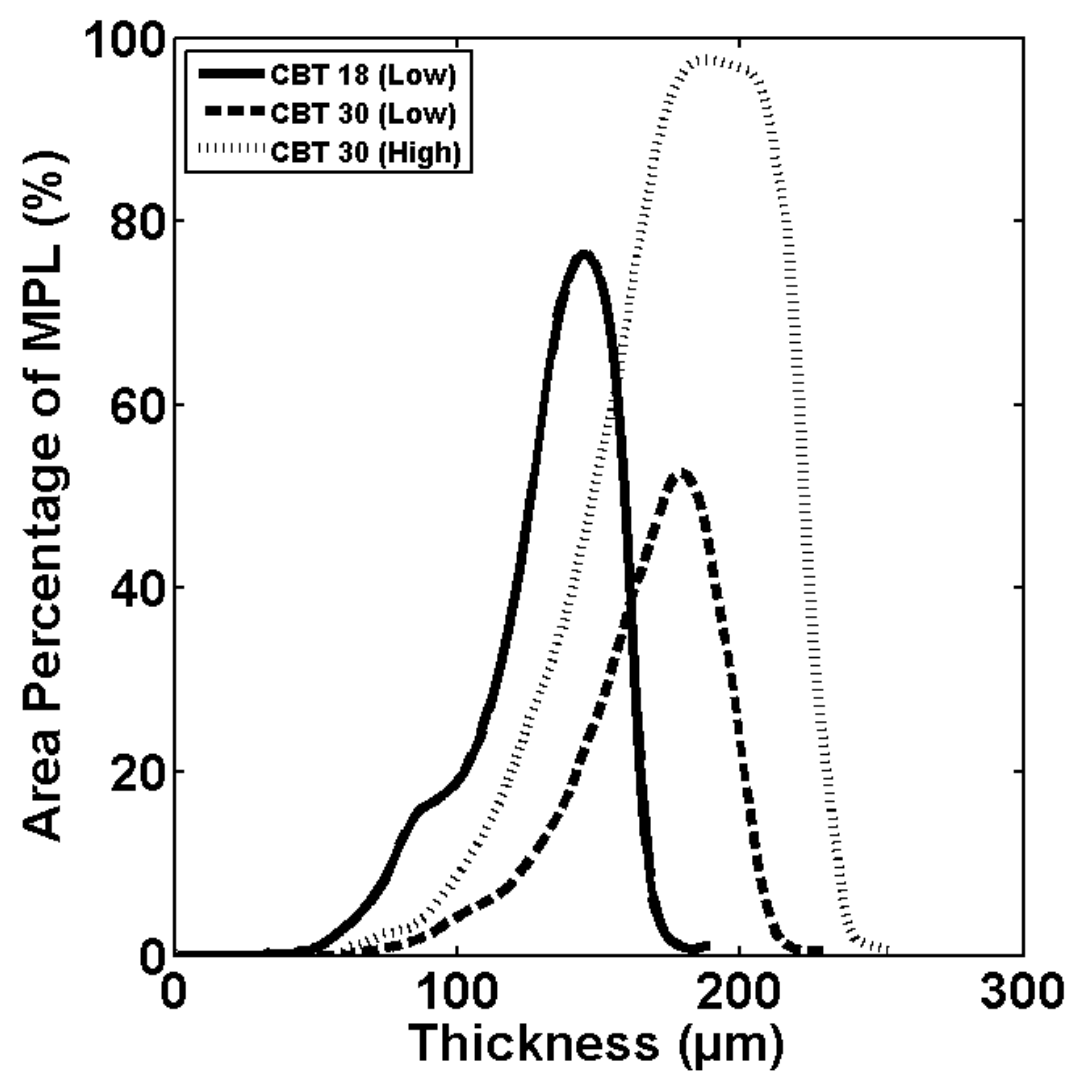

